\title{
Mode-scrambling security using short pulses in multimode graded-index fiber
}

\author{
Mario Zitelli \\ Department of Information \\ Engineering, Electronics and \\ Telecommunications (DIET) \\ Sapienza University of Rome \\ Via Eudossiana 18, 00184 Rome, Italy \\ mario.zitelli@uniroma1.it \\ Fabio Mangini \\ Department of Information \\ Engineering \\ University of Brescia \\ Via Branze 38, 25123 Brescia, Italy \\ fabio.mangini@unibs.it
}

\author{
Mario Ferraro \\ Department of Information \\ Engineering, Electronics and \\ Telecommunications (DIET) \\ Sapienza University of Rome \\ Via Eudossiana 18, 00184 Rome, Italy \\ mario.ferraro@uniroma1.it \\ Stefan Wabnitz \\ Department of Information \\ Engineering, Electronics and \\ Telecommunications (DIET) \\ Sapienza University of Rome \\ Via Eudossiana 18, 00184 Rome, Italy \\ stefan.wabnitz@uniromal.it
}

\begin{abstract}
Secure optical transmission based on random modal coupling in multimode fibers is analyzed using ultrashort pulses. The effects of modal dispersion over groups of degenerate modes is studied, and the compatibility of soliton spatial-division-multiplexing (SDM) with modal-scrambling based security is assessed.
\end{abstract}

Keywords-multimode fibers, random mode coupling, security.

\section{INTRODUCTION}

Multimode fibers (MMFs) offer the potential for increasing the transmission capacity of long-distance optical links via space-division-multiplexing (SDM), which exploits the multiple transverse modes of the fiber as information carriers. Graded-index (GRIN) MMFs are capable of of supporting the propagation of MM solitons over long distances [1-3]. Optical solitons in fibers have been extensively and successfully studied over the past fifty years, leading to significant progress in long-distance optical communications and mode-locked lasers [4,5]. Although nearly all of these investigations involved the generation and propagation of singlemode fiber solitons, optical solitons can be supported by multimode optical fibers (MMFs) as well [6-8].

It was previously assessed [9] that random mode coupling (RMC), in multimode fibers, can be conveniently used for implementing a transmission security at the physical layer, scrambling the modes in a way that only the legitimate receiver (Bob) is able to recover. The illegitimate receiver (Eve) will not be able to reconstruct the message sent by Alice, even in the worst-case in which she listens during the channel calibration procedure. In a second work, it was demonstrated the possibility to implement highdimensional quantum networks in multimode fibers supporting modes with optical angular momentum (OAM), similar to Laguerre-Gauss modes in GRIN fibers.

In this work, we demonstrate by numerical simulations, that spatio-temporal soliton transmission in GRIN fiber is compatible with both SDM and with RMC-based secure transmission. The former property is promising for implementing robust, high optical signal-to-noise ratio
(OSNR) SDM transmission in GRIN fibers; the latter property permits to obtain physical layer security based on modal scrambling into each transmitted SDM channel.

\section{MODE SCRAMBLING SECURITY}

A commercial multimode fiber is able to transmit hundreds of modes with same (degenerate) or different (nondegenerate) group velocity. In the assumption of weak random coupling [11], the degenerate modes randomly exchange power between them. Moreover, at high pulse energy, nonlinear coupling through inter-modal four-wave mixing (IM-FWM) and stimulated Raman scattering (IMSRS) is responsible for power transfer among nondegenerate or degenerate modes [12]. Waveform shaping [13], commonly produced using spatial light modulators (SLM) at the transmitter, may be used for properly tailoring the modal content at Alice's side, in order to reproduce a given modal pattern at Bob's output; this will result into a well established speckle pattern that Bob may recognize as a calibration code. The illegitimate recipient Eve will receive a modal pattern resulting from the random mode scrambling of only a portion of the fiber; this feature is advantageously used to hide information to Eve during the calibration phase. Such a method does not require single-photon transmission, like in quantum information case, and it is therefore compatible with high pulse energy, high capacity transmission.

Following this method, the calibration phase between Alice and Bob must invert the following equation:

$$
\bar{y}_{B}=T \cdot \bar{x}_{A}+n_{B}
$$

where $\bar{x}_{A}, \bar{y}_{B}$ are the $N$ mode distributions at Alice and Bob sides respectively, and $T$ the transmission matrix; $n_{B}$ is the received noise. The ideal calibration procedures consists in finding, and applying at the transmitter, an inverse matrix $T^{\prime}$ such as

$$
\bar{y}_{B}=T \cdot T^{\prime} \cdot \bar{x}_{A}+n_{B}=\bar{x}_{A}+n_{B} .
$$


Using the inverse matrix at the transmitter, Alice is able to reproduce the transmitted modal combination at Bob's side, which doesn't need to use an inverse matrix.

At Eve's side, the received mode combination will be given by

$$
\bar{y}_{E}=S \cdot T^{\prime} \cdot \bar{x}_{A}+n_{E} \quad,
$$

with $S$ the transmission matrix from Alice to Eve. The matrix inversion is necessary to Eve, resulting into a scrambling of the noise term, i.e.

$$
\bar{y}_{E}^{\prime}=\bar{x}_{A}+\left(S \cdot T^{\prime}\right)^{-1} n_{E}
$$

By transmitting an arbitrary noise, it results the production of arbitrarily amplified noise at Eve's side, considerably reducing the optical signal-to-noise factor (OSNR). It is possible to deliver virtually unreadable information to Eve.

The advantage of this method is the possibility to transmit high power pulses carrying the modal information; soliton transmission can be adopted in order to reduce nonlinear impairments and increase the channel capacity.

In the next section, it will be shown that the mode scrambling method is compatible with SDM and multimode soliton transmission.

\section{SOLITON SDM IN GRIN FIBERS}

In recent studies [14,15], we demonstrated that a new class of optical solitons, clamped to the modal walk-off length, propagate in GRIN fibers with fixed energy and pulsewidth, that depend only on the input pulse wavelength, and are independent on the input pulse duration. At telecom wavelengths, $1550 \mathrm{~nm}$, a spatiotemporal soliton including non-degenerate modes always forms with fixed, 120 fs pulsewidth, and 2-3 nJ energy. This class of walk-off solitons is of course of no interest for telecom applications; by the way, it poses the basis for a stable long-haul SDM transmission.

Figure 1 shows the numerical example of a 10 ps pulse launched at $1550 \mathrm{~nm}$ on a GRIN fiber span of $5 \mathrm{~km}$, stimulating at input the first 15 modes (Laguerre-Gauss modes $\mathrm{LP}_{01}$ to $\left.\mathrm{LP}_{03}\right)$.

Simulations are performed by means of a coupled-mode equation model $[16,17]$, modified to include wavelengthdependent losses and random mode coupling. Second and third order chromatic dispersion, as well as modal dispersion, are included. The fields of the propagating modes are also coupled by inter-modal FWM and SRS via mixing products of the type $Q_{p l m n} A_{l} A_{m} A_{n}{ }^{*}$, where $Q_{p l m n}$ are coupling coefficients proportional to the overlap integrals of transverse modal field distributions.

The left inset of Fig. 1 is the input pulse, with energies:

$\mathrm{E}_{1}=0.05 \mathrm{~nJ}$ for the mode $\mathrm{LP}_{01}$.

$\mathrm{E}_{2}=0.075 \mathrm{~nJ}$ equi-distributed for the degenerate modes $\mathrm{LP}_{11 \mathrm{a}}, \mathrm{LP}_{11 \mathrm{~b}}$.

$\mathrm{E}_{3}=0.1 \mathrm{~nJ}$ distributed among the degenerate modes $\mathrm{LP}_{21 \mathrm{a}}$, $\mathrm{LP}_{21 b}, \mathrm{LP}_{02}$.

$\mathrm{E}_{4}=0.15 \mathrm{~nJ}$ among the degenerate modes $\mathrm{LP}_{31 \mathrm{a}}, \mathrm{LP}_{31 \mathrm{~b}}, \mathrm{LP}_{12 \mathrm{a}}$, LP12b.

$\mathrm{E}_{5}=0.25 \mathrm{~nJ}$ among the degenerate $\mathrm{LP}_{41 \mathrm{a}}, \mathrm{LP}_{41 \mathrm{~b}}, \mathrm{LP}_{22 \mathrm{a}}, \mathrm{LP}_{22 \mathrm{~b}}$, $\mathrm{LP}_{03}$.
Right inset is the obtained output pulse pattern after $5 \mathrm{~km}$, setting a negligible RMC. It is shown that, by properly choosing the modal power distribution, 5 soliton pulses are obtained at output, one for each group of degenerate modes. The modes being degenerate, it is possible to obtain a $10 \mathrm{ps}$ soliton for each degenerate group; by the way, the 5 solitons cannot merge together to form a single soliton with nondegenerate modes, because only 120 fs solitons are permitted in that case.

This particular property permits the transmission of stable and independent soliton modal channels (5 in the example), each composed by several degenerate modes; the solitons perform collisions between them but are not allowed to merge. Moreover, transmission is obtained at high channel power, that may arrive to hundreds of $\mathrm{mW}$ per modal channel, increasing the capacity both as a consequence of the use of SDM, and by the improved OSNR.

Figure 2 represents the same case illustrated in Fig. 1, including a moderate RMC between degenerate modes (weak modal coupling [11]); Figure 3 is the case with a strong amount of RMC among degenerate modes; the left insets are the modal energies vs. distance; the right insets are the output mode powers.

In both cases of moderate and strong RMC, modal energy is exchanged between degenerate modes, not randomly by means of the nonlinear coupling, and also randomly by means of the RMC. Soliton transmission for each group of modes results, by the way, unperturbed by the random modal scrambling into each group of modes.

\section{CONCLUSIONS}

The possibility of propagating multiple soliton pulses in a GRIN fiber, one for each group of degenerate modes, unaffected by the RMC and the nonlinear coupling, appears of particular interest when considering the possibility to exploit the intrinsic modal scrambling induced by the RMC, in order to add security to each transmitted modal channel. The propagated solitons appear temporally undistorted and conserve their pulsewidth (10 ps at $1550 \mathrm{~nm}$, in the example of section III), despite of the continuous modal scrambling into each pulse; pulsewidth is preserved, and the amount of spectral red-shift produced by Raman soliton self-frequency shift appears negligible.

Modal power is very high in the considered example (hundreds of $\mathrm{mW}$ per wavelength), demonstrating the possibility of implementing high capacity modal transmission. Moreover, the illustrated transmission appears compatible with both soliton SDM and wavelength-division multiplexing (WDM).

As it was seen in section II, the high OSNR at Bob's side does not imply that an illegitimate recipient is able to read a clear transmission. Eve's side is affected by optical noise induced by the partial modal scrambling over the Alice-Eve fiber segment; Eve's OSNR may be reduced by a proper choice of the noise at the transmitter, that will be scrambled together with the modes power by Eve's efforts to invert the transmission matrix. 


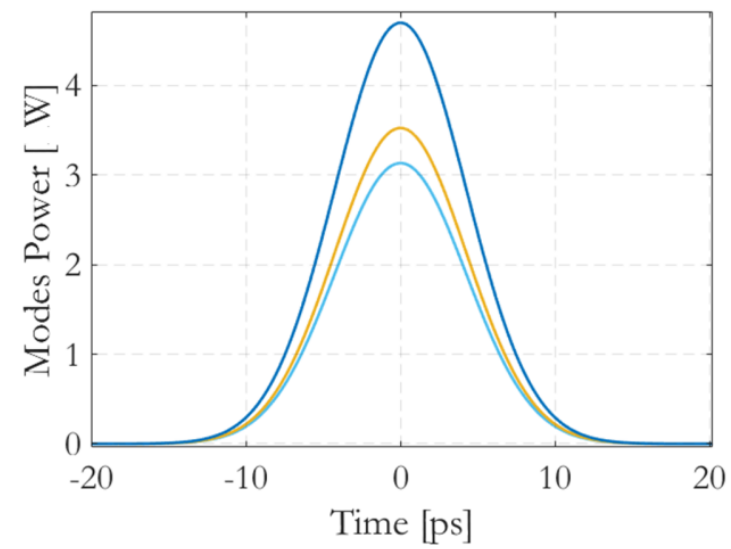

Fig. 1 - Numerical simulation of transmitted 10 ps pulse, 15 modes, 1550 $\mathrm{nm}$, at input (left) and after $5 \mathrm{~km}$ of GRIN fiber (right). Mode power distribution at input is described in the main text. No RMC is included.

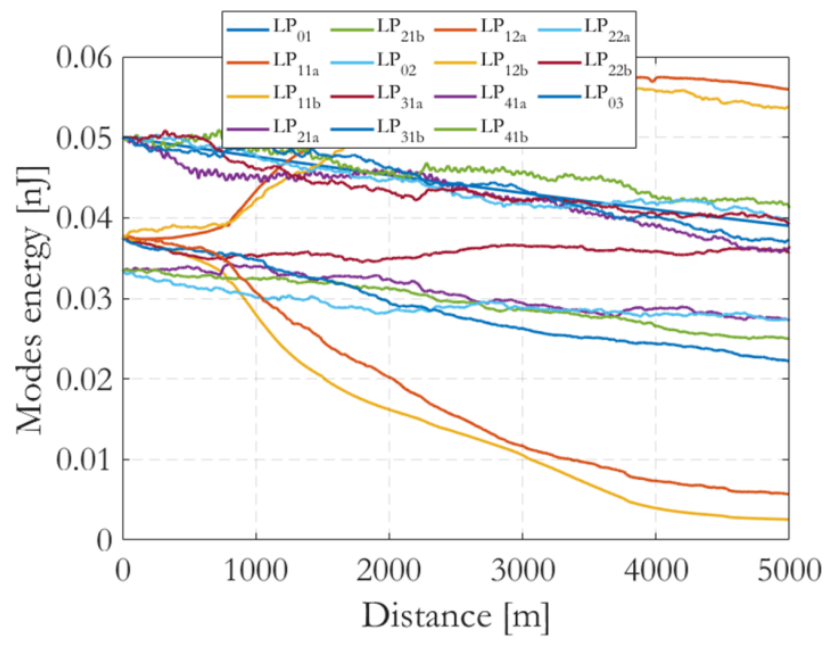

Fig. 2 - Numerical simulation of Fig. 1, including moderate RMC. Left modal energy vs. distance; right: output modal power.

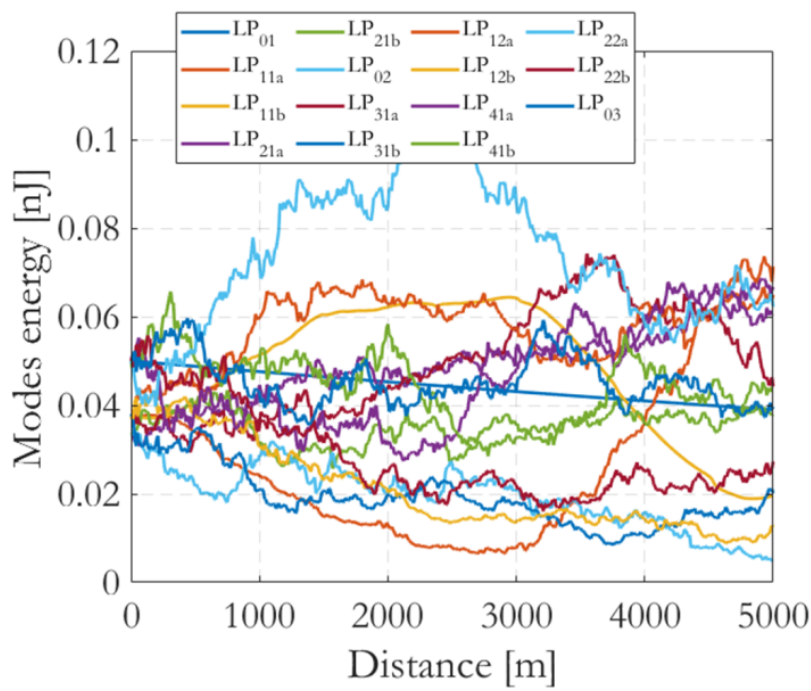

Fig. 3 - Numerical simulation of Fig. 1, including strong RMC. Left: modal energy vs. distance; right: output modal power.
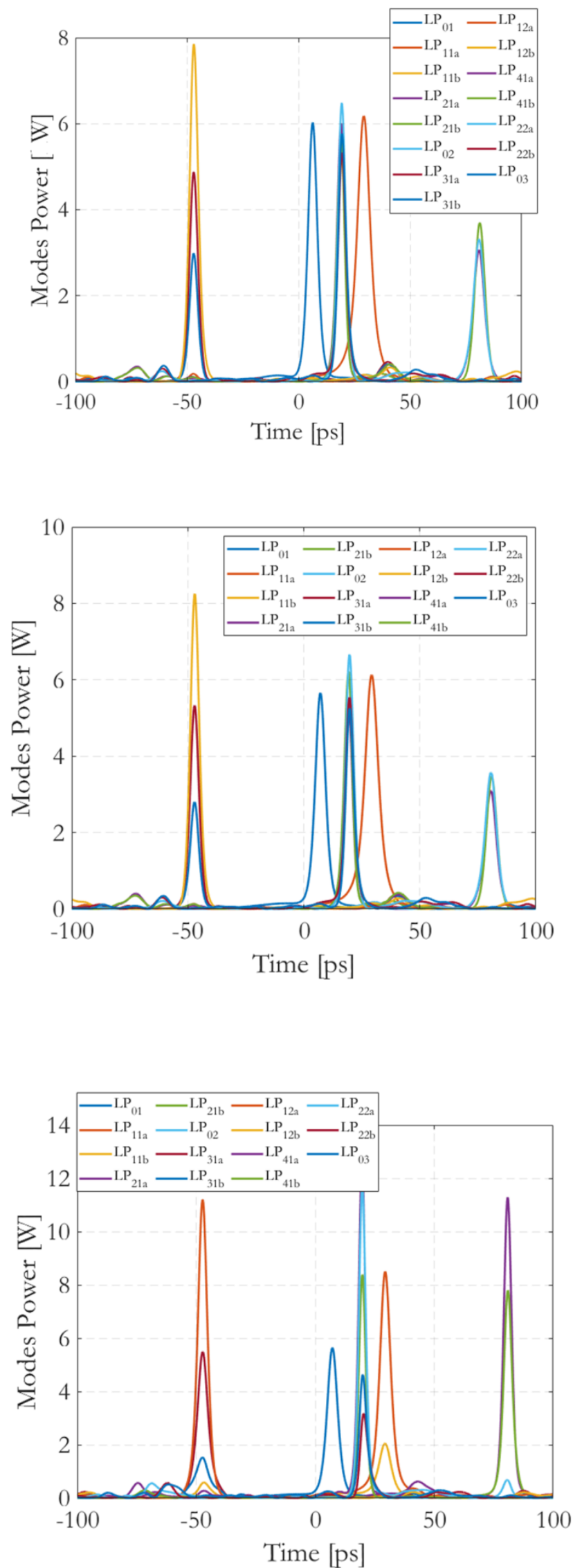


\section{REFERENCES}

[1] B. Crosignani and P. D. Porto, "Soliton propagation in multimode optical fibers," Opt. Lett., vol. 6, pp. 329-330, 1981.

[2] A. S. Ahsan and G. P. Agrawal, "Graded-index solitons in multimode fibers," Opt. Lett., vol. 43, pp. 3345-3348, 2018.

[3] Renninger, W. H., \& Wise, F. W., Optical solitons in graded-index multimode fibres, Nat. Commun., vol. 4, pp. 1719, 2013.

[4] A. Hasegawa and M. Matsumoto, Optical Solitons in Fibers (Springer-Verlag Berlin Heidelberg, 2003).

[5] V. E. Zakharov and S. Wabnitz, Optical Solitons: Theoretical Challenges and Industrial Perspectives (Springer-Verlag Berlin Heidelberg, 1999).

[6] A. Hasegawa, "Self-confinement of multimode optical pulse in a glass fiber,” Opt. Lett. 5, 416-417 (1980).

[7] B. Crosignani, A. Cutolo, and P. D. Porto, "Coupled-mode theory of nonlinear propagation in multimode and single-mode fibers: envelope solitons and self-confinement," J. Opt. Soc. Am. 72, 1136-1141 (1982).

[8] K. Krupa, A. Tonello, A. Barthélémy, T. Mansuryan, V. Couderc, G. Millot, P. Grelu, D. Modotto, S. A. Babin, and S. Wabnitz, "Multimode nonlinear fiber optics, a spatiotemporal avenue," APL Photonics 4, 110901 (2019).

[9] S. Rothe1, N. Koukourakis, H. Radner, A. Lonnstrom, E. Jorswieck, J. W. Czarske1, "Physical Layer Security in Multimode Fiber Optical Networks", Scient. Reports 10:2740 (2020).

[10] D. Cozzolino, D. Bacco, B. Da Lio, K. Ingerslev, Y. Ding, K. Dalgaard, P. Kristensen, M. Galili, K. Rottwitt, S. Ramachandran, L. Katsuo Oxenløwe1, "Orbital Angular Momentum States Enabling Fiber-based High-dimensional Quantum Communication”, Phys. Rev. Appl. 11, 064058 (2019).

[11] S. Mumtaz, R. J. Essiambre, G. P. Agrawal, "Nonlinear Propagation in Multimode and Multicore Fibers: Generalization of the Manakov Equations", JLT 31(3), pp. 398-406, 2012

[12] L. G. Wright, W. H. Renninger, D. N. Christodoulides, F. W.Wise, "Spatiotemporal dynamics of multimode optical solitons", Opt. Expr. 23(3), pp. 3492-3506, 2015.

[13] I. N. Papadopoulos, S. Farahi, C. Moser, D. Psaltis, "Focusing and scanning light through a multimode optical fiber using digital phase conjugation", Opt. Express 20, pp. 10583-10590 (2012).

[14] M. Zitelli, M. Ferraro, F. Mangini, S. Wabnitz, "Single-mode spatiotemporal soliton attractor in multimode GRIN fibers," Phot. Research, vol.9, no. 5, pp. 741-748, 2021

[15] M. Zitelli, F. Mangini, M. Ferraro, O. Sidelnikov, S. Wabnitz, "Conditions for walk-off soliton generation in a multimode fiber", Comm. Phys., in press.

[16] F. Poletti and P. Horak, "Description of ultrashort pulse propagation in multimode optical fibers," J. Opt. Soc. Am. B vol. 25(10), pp. 1645-1654, 2008.

[17] L. G. Wright, Z. M. Ziegler, P. M. Lushnikov, Z. Zhu, M. A. Eftekhar, D. N. Christodoulides, and F.W. Wise, "Multimode nonlinear fiber optics: Massively parallel numerical solver, tutorial, and outlook," IEEE J. Sel. Top. Quantum Electron., vol. 24, pp. 1$16,2018$. 